\title{
Caracterización y propuesta de recuperación de aplacado de mortero polimérico de la parroquia de San Pablo de Sevilla
}

\section{Characterization and recovery proposal of polymer mortar coating plates of the parish of San Pablo in Seville}

$\underline{\text { V. Flores-Alés }}^{(*)}$, F. J. Alejandre ${ }^{(*)}$, F. J. Blasco-López ${ }^{(*)}$, J. M. Macías-Bernal ${ }^{(*)}$

\begin{abstract}
RESUMEN
El presente trabajo aborda el estudio de los elementos de fachada, fabricados con mortero polimérico, de la parroquia de San Pablo de Sevilla (1961). Se ha llevado a cabo la caracterización de las placas de revestimiento y el análisis de las posibles vías de recuperación del material alterado con el objeto de propiciar una futura intervención. El estudio de las resinas empleadas como aglutinante, identificadas como un poliéster alifático, permite aproximar la compatibilidad de los productos de restauración seleccionados. La caracterización aporta información sobre la capacidad estructural y mejora de propiedades conseguida. El control colorimétrico es una necesidad inexcusable, puesto que cualquier actuación sobre la superficie de las piezas conlleva una modificación cromática. Los análisis y ensayos han permitido establecer un sistema de tratamiento adecuado para la recuperación de las placas y aportar la información suficiente a los responsables del proyecto de intervención para una toma de decisiones justificada.
\end{abstract}

Palabras clave: mortero polimérico; resina; aplacado; restauración.

\section{ABSTRACT}

This paper deals with the study of the facade elements of the parish of San Pablo in Seville (1961). They are made with polymer mortar. A characterization of the coating plates was carried out and possible solutions for recovering the altered material were analyzed in order to facilitate a future intervention. The resins used as a binder were identified as aliphatic polyester. The resin study was carried out to assure the compatibility of restoration products. Materials characterization provides information about the structural capacity of the plates and the properties achieved. The plates' colorimetric control is an inexcusable necessity, since any action on the surface of the pieces leads to a color change. All the analyses and testing carried out have led to the creation of an appropriate treatment system for recovery of the plates that provides sufficient information to the intervention project managers to justify decision-making.

Keywords: polymer mortar; resin; coating plates; restoration.

(*) Departamento de Construcciones Arquitectónicas II, Universidad de Sevilla, Sevilla (España). Persona de contacto/Corresponding author: vflores@us.es (V. Flores-Alés) ORCID: http://orcid.org/oooo-0oo3-4329-0020 (V. Flores-Alés); http://orcid.org/oooo-00o3-0942-8313 (F. J. Alejandre); http://orcid.org/oooo-0002-0488-3061 (F. J. Blasco-López); http://orcid.org/oooo-0001-6073-9745 (J. M. Macías-Bernal)

Cómo citar este artículo/Citation: Flores-Alés, V., Alejandre, F. J., Blasco-López, F. J., Macías-Bernal, J. M. (2017). Caracterización y propuesta de recuperación de aplacado de mortero polimérico de la parroquia de San Pablo de Sevilla. Informes de la Construcción, 69(546): e194, doi: http://dx.doi.org/10.3989/ic.16.026.

Copyright: (C) 2017 CSIC. Licencia / License: Salvo indicación contraria, todos los contenidos de la edición electrónica de Informes de la Construcción se distribuyen bajo una licencia de uso y distribución Creative Commons Attribution License (CC BY) Spain 3.o. 


\section{INTRODUCCIÓN}

La iglesia de San Pablo de Sevilla es un edificio propiedad del arzobispado, proyectado en 1961 por los arquitectos Luis Recasens Méndez y Antonio de la Peña Neila. El templo, de planta ligeramente trapezoidal con una capilla adosada, se construye como equipamiento del barrio A del Polígono de San Pablo levantado en el marco del primer Plan Desarrollo (1964-1967). La estructura se conforma por un sistema de jácenas de hormigón apoyadas sobre estructura metálica, dando lugar a un espacio absolutamente funcional y desornamentado, en el que destacan las vidrieras que conforman un curioso sistema de iluminación junto con lucernarios en la cubierta, compuestos de bastidor metálico con vidrio armado. Esta construcción se puede incluir dentro de una interesante muestra de búsqueda de renovación tipológica, que tiene como resultado algunos de los mejores ejemplos de la arquitectura de los años 60 en Sevilla (Figura 1) (1) (2) (3). Actualmente tiene asignado un grado de protección B (4), en el que se encuentra protegida la totalidad del conjunto, permitiéndose intervenciones de conservación y mantenimiento y actuaciones interiores que no afecten al sistema estructural, composición espacial, estructura ni cubiertas, según se indica en el PGOU de Sevilla (hoja E, 14-13).

El revestimiento de la fachada, que es el elemento que nos ocupa, está compuesto de un sistema de aplacado con piezas prefabricadas de mortero polimérico de apariencia unimodu- lar, lo que da lugar a un efecto homogéneo de rugosidad y ligereza en las placas. Dicho revestimiento se encuentra adherido sobre un muro de fábrica de ladrillo de un pie (Figura 2).

Las condiciones del entorno no son particularmente desfavorables, ni por acción de tráfico ni por humedad excesiva; pero el paso de tiempo, la incidencia de la radiación UV y los naturales ciclos térmicos e higrométricos, con períodos de fuerte calentamiento propios del verano en Sevilla, han dado como resultado el lógico deterioro de la resina polimérica empleada como aglutinante del árido en el aplacado, si bien no es posible discernir el efecto independiente que cada uno de ellos ha causado; el estado de conservación de las cuatro fachadas difiere en función de la orientación, por lo que se entiende que las condiciones ambientales han propiciado una evolución ligeramente distinta en la degradación del material. El comportamiento de estos morteros y los efectos de las condiciones de exposición en morteros de matriz polimérica ha sido estudiado por diversos autores (5) (6) (7) (8) (9) (10) (11). En este sentido, los investigadores evalúan propiedades como contenido en resinas, estabilidad térmica, comportamiento mecánico, degradación UV, estabilidad de color..., a partir de ello, y en función del objetivo del estudio, se han seleccionado los ensayos y determinaciones para la caracterización del material.

Debe reseñarse la importancia del buen criterio establecido por los técnicos responsables del proyecto de restauración de

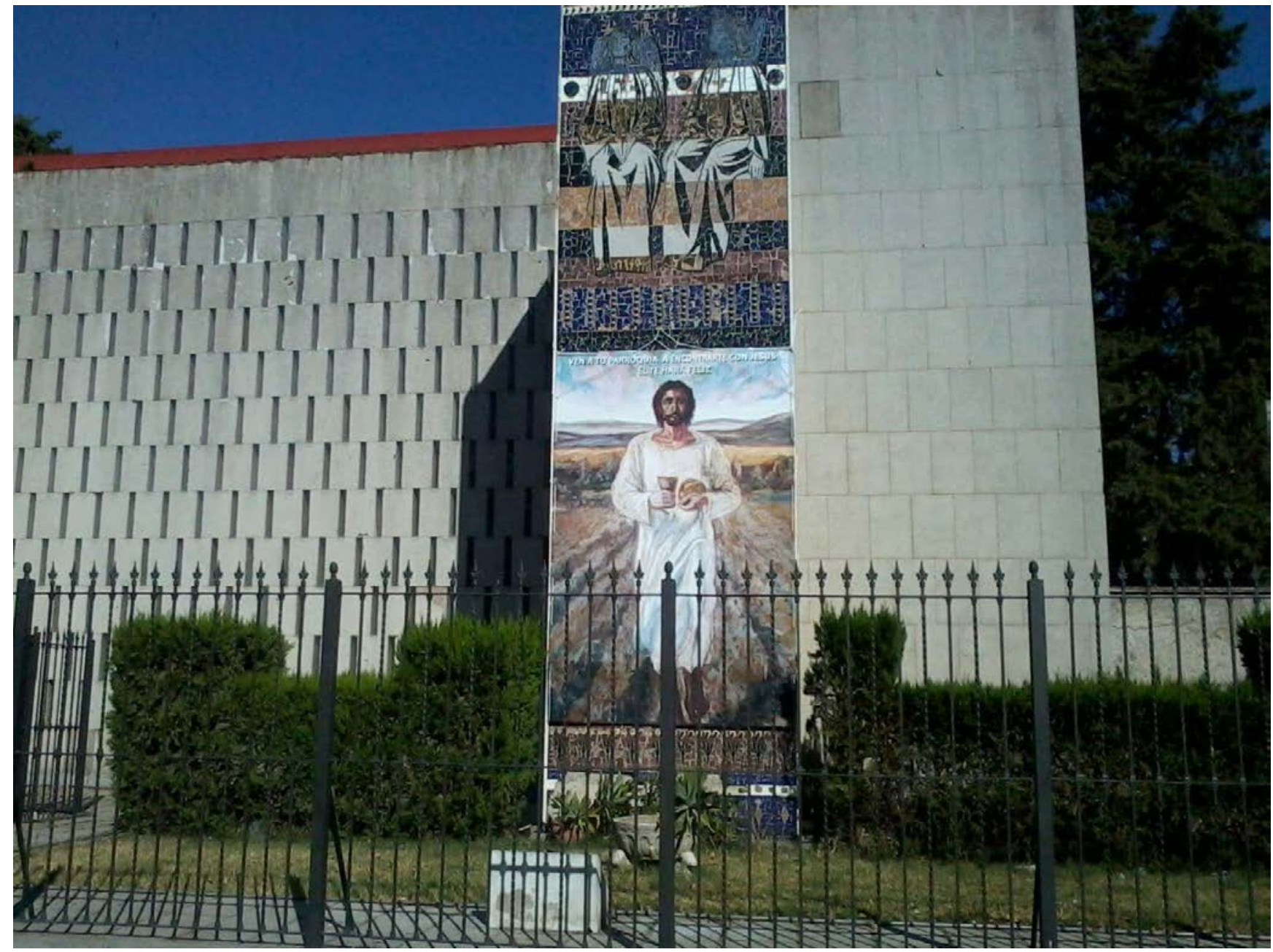

Figura 1. Imagen exterior del templo de San Pablo (Sevilla). 


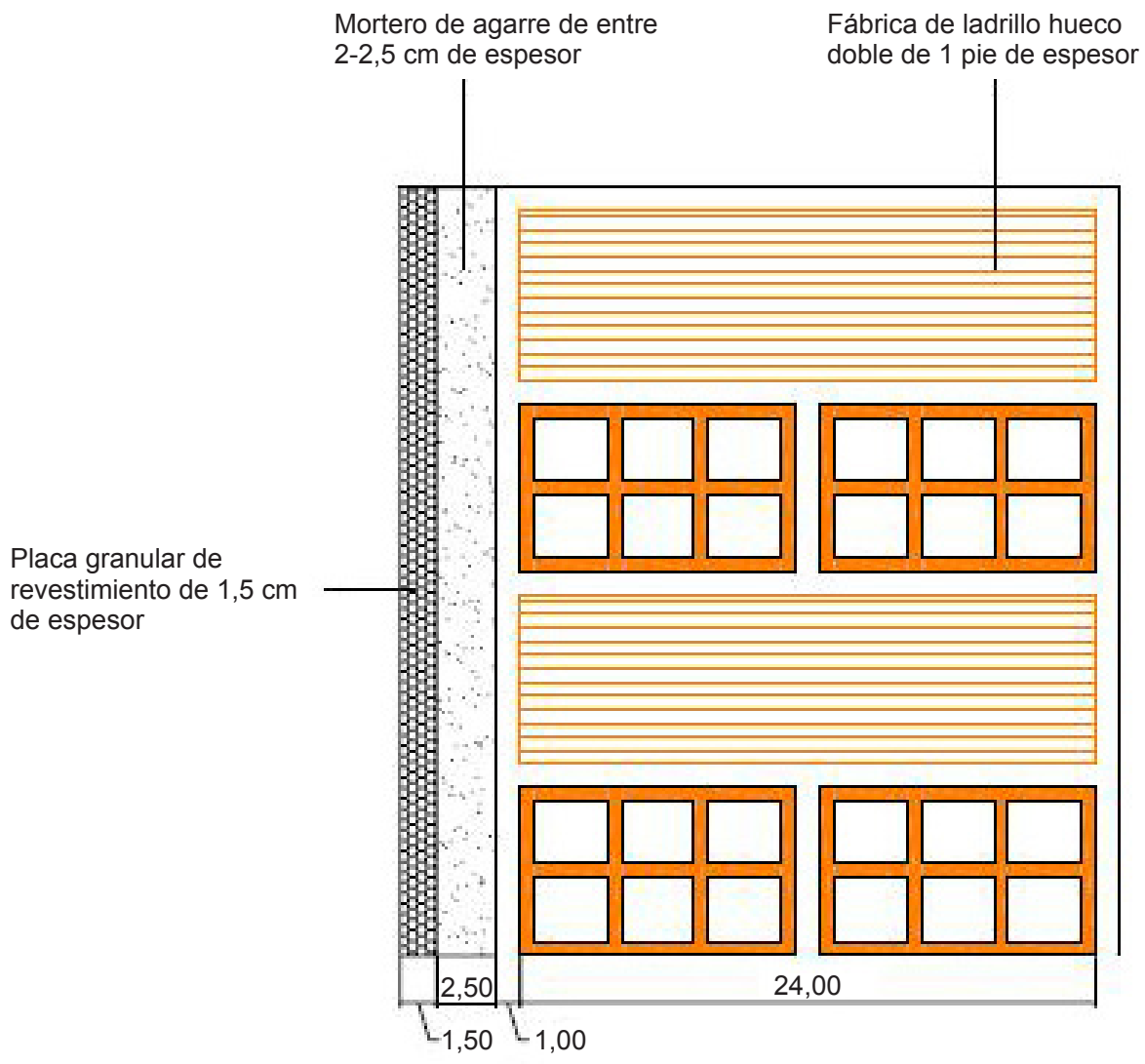

Cotas en $\mathrm{cm}$

Escala 1:50

Figura 2. Detalle de la sección de la fábrica, mortero de agarre y aplacado.

la fachada del templo objeto de estudio, considerando el interés de la conservación del aplacado original que forma parte de un edificio representativo de la arquitectura de su época y cuyo valor no debe despreciarse por el hecho de tratarse de una edificación contemporánea. La intervención de conservación es incuestionable frente a la posibilidad de sustituir el elemento existente por un acabado aparentemente similar, como sería un mortero monocapa, que pervertiría el proyecto original alterando un elemento clave del diseño y la estética del templo.

Llegado este punto se hace necesaria la caracterización de las placas de revestimiento y el análisis de las posibles vías de recuperación del material alterado con el objeto de propiciar una futura intervención que permita dar respuesta al proceso de deterioro creciente que está experimentando el exterior del templo.

\section{OBJETIVO}

El objetivo del presente trabajo es caracterizar los materiales que componen la placa del revestimiento, determinando la composición de la resina conglomerante, las propiedades físicas y mecánicas más determinantes de las piezas para evaluar su estado de conservación y la colorimetría. A partir de este punto se ha analizado la eficacia adhesiva y los efectos producidos por los posibles tratamientos a aplicar sobre el aplacado existente, mediante una selección previa de productos compatibles que guarden la debida similitud con las resinas utilizadas originalmente, con la finalidad de aportar información a un futuro proyecto de intervención que garantice la solidez de las piezas y la neutralidad cromática, al tiempo que, por estudios previos, garantizan una adecuada durabilidad (12) (13) (14).

\section{MATERIALES Y MÉTODO}

\subsection{Materiales}

Se tomaron muestras del aplacado del edificio bajo la asesoría y supervisión de un restaurador y un técnico del Arzobispado, en distintas fachadas y en función del estado de conservación y fijación de las placas (Figura 3). Se debe señalar, con relación al estado de conservación, que si bien la cohesión en la zona interior de las placas es buena e independiente a la orientación de las fachadas (menor degradación de las resinas), sí muestran una gran fragilidad. Sí se perciben diferencias en el grado de desprendimiento de los áridos superficiales entre las fachadas norte-oeste (más sombrías) y las sur-este (más soleadas). Este contraste puede estar originado por la fuerte insolación que se da en las fachadas sur-este, lo que ha provocado un mayor deterioro superficial de la resina (9), por lo que el desprendimiento del árido es mucho más evidente. Esta circunstancia determina el plan de ensayos descrito en la metodología (Tabla 1).

Con el objeto de disponer de árido original para la realización de ensayos, se recogió del perímetro del edificio un volumen 

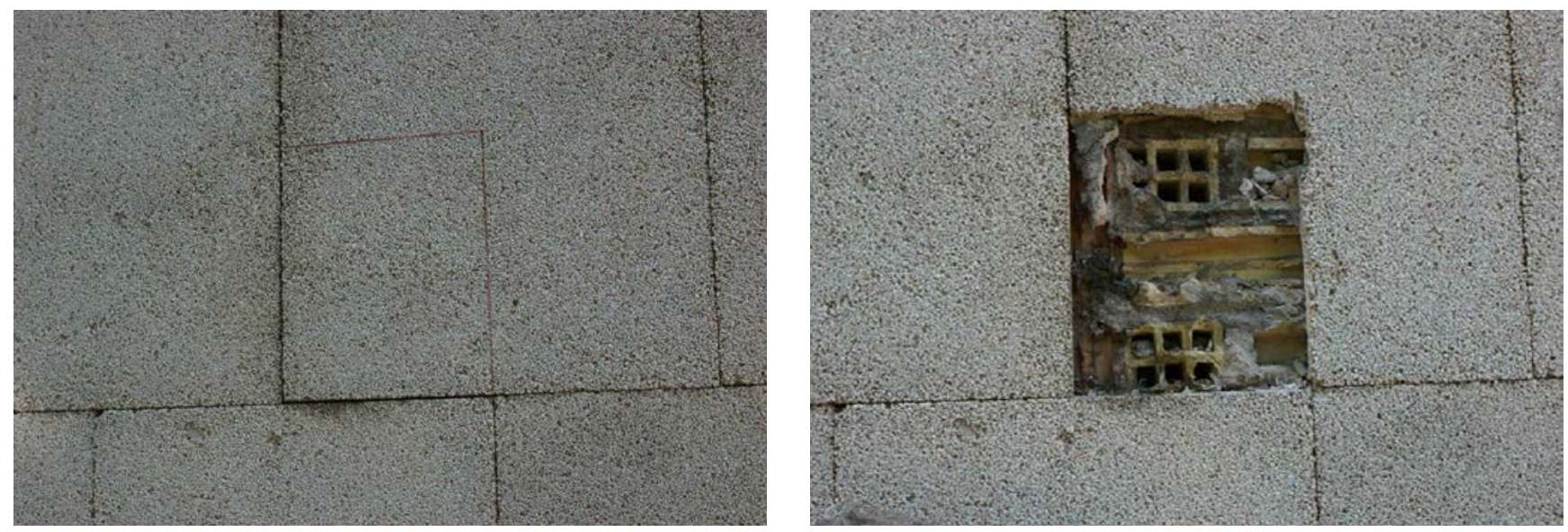

Figura 3. Toma de muestras del aplacado.

suficiente de agregado desprendido, sometiéndose éste a un proceso de limpieza mediante soplado, flotación y posterior desecación. El reconocimiento visual del árido, apoyado por instrumental de aumento, permite clasificarlo petrográficamente como un mármol machacado o marmolina, no presentando indicios de alteración química ni física, las imágenes tomadas con lupa de aumento permiten comprobar en la cara exterior de la placa las superficies inalteradas de los granos de marmolina, así como los restos de la resina que los mantienen unidos, por el contrario en la cara interior se observa cómo la resina recubre completamente los granos, compactándolos y aislándolos (Figura 4). Con este árido se realizó un ensayo de carácter organoléptico para ver el efecto que sobre el árido suelto producía la pulverización de los productos consolidantes.

Con el objeto de comparar el polímero originalmente utilizado en las piezas de la parroquia de San Pablo con resinas comerciales actuales empleadas para la restauración y fabricación de morteros poliméricos, se realizaron también espectros sobre una selección de productos comerciales indicados por el equipo de restauradores, siendo éstos: Acrisil201 (resina acril-silicónica), Acryl33 (resina acrílica), BA2028, Epo15o (resina epoxídica), Fluoline (elastómero fluorado + polímero acrílico) y ParaloidB72 (resina metiletilacrílica).

\subsection{Metodología de caracterización de las piezas de revestimiento}

La resina polimérica utilizada originalmente en la fabricación de las placas se estudió a partir de su extracción con acetona y posterior cuantificación e identificación del tipo de compuesto orgánico mediante FTIR (espectroscopia infrarroja mediante transformada de Fourier) con un espectrofotómetro Jasco FT/IR4100 sobre un rango $4.000-400 \mathrm{~cm}^{-1}$. Este aparato está equipado con accesorio ATR MIRacleTM para medidas de reflexión total atenuada (ATR), esto permite la medida directa de las muestras sin necesidad de disolventes o medios de dispersión que generan «zonas ciegas» en el espectro. Los espectros se obtuvieron a partir de 70-90 barridos.

Se ha realizado el estudio óptico de las superficies de las placas con microscopio stereozoom marca LEICA, modelo S8 APO, con cámara de captura LEICA DC300 y software IM50 (Image Manager) v.1.20.

El estudio de la granulometría del árido de las placas se ha realizado con el material original desprendido de las placas, una vez lavado y limpio se tamizó empleando la serie de tamices: 0,063 mm, 0,125 mm, 0,25 mm, 0,5 mm, $1 \mathrm{~mm}, 2 \mathrm{~mm}$, $4 \mathrm{~mm}, 8 \mathrm{~mm}, 16 \mathrm{~mm}, 31,5 \mathrm{~mm}$ y $63 \mathrm{~mm}$, correspondientes a la Norma UNE EN 933-2 (15).

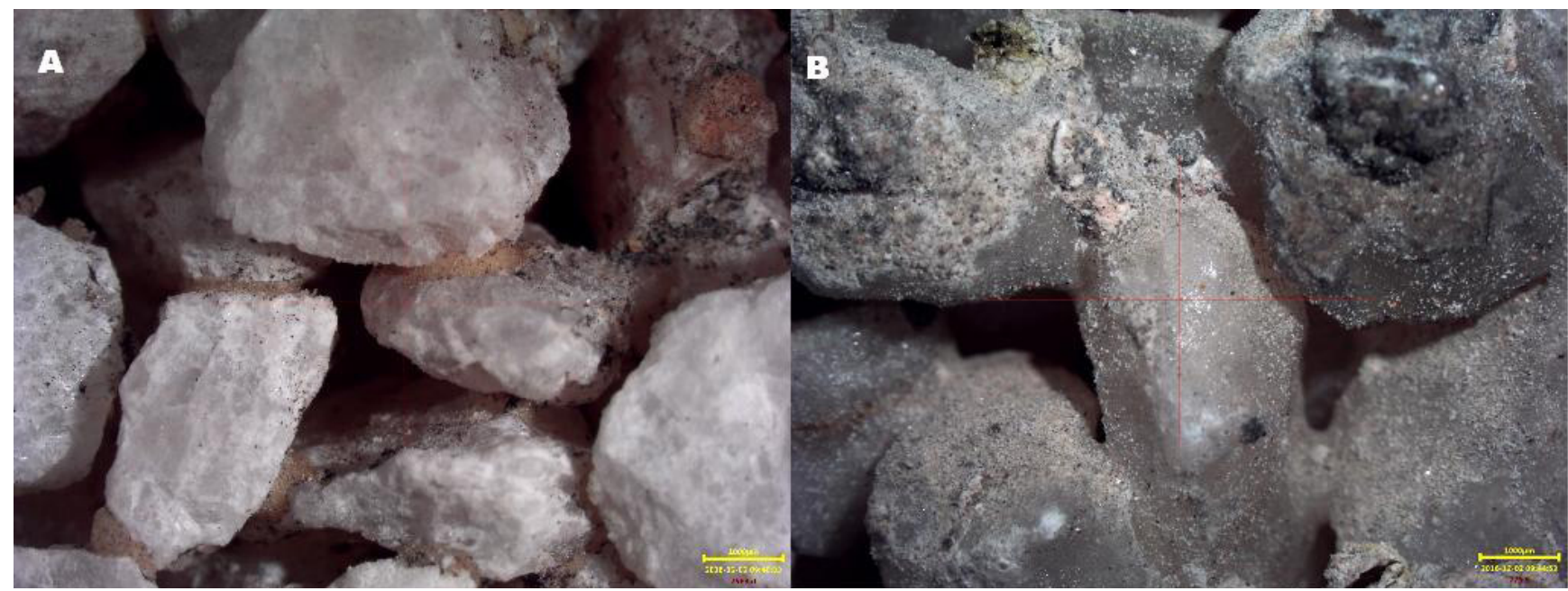

Figura 4. A) Imagen de los granos de árido en la cara exterior entre los que se aprecian los restos de resina de color ámbar. B) Imagen de los granos en la cara interior de la placa, apreciándose los restos de cemento del mortero de agarre. 
La caracterización física y mecánica se ha realizado sobre dos muestras, la selección de las mismas ha tenido por objeto minimizar al máximo el daño sobre las fachadas del edificio, considerando las diferencias existentes en el estado de conservación (tal como se ha indicado en el apartado 3.1), se ha estimado suficiente extraer una la fachada norte-oeste y otra de la sur-este.

Las propiedades físicas que se han determinado en las placas (PSP-1, PSP-2) son la densidad aparente, el coeficiente de absorción y la porosidad accesible al agua, caracterizándose todas ellas por suministrar información sobre la estructura del material. Los métodos seguidos para la determinación de estas propiedades se basan en la determinación geométrica sobre probetas y saturación con agua a presión atmosférica de acuerdo con la norma UNE-EN-13755:2008 (16) y sometida a vacío según norma UNE-EN-1936:2007 (17).

La caracterización mecánica se ha llevado a cabo mediante el ensayo de resistencia a flexión de las placas según la norma UNE-EN-12372:2006 (18), que especifica un método de ensayo para la determinación de la resistencia a flexión bajo carga concentrada para piedra natural (19). Se ha realizado sobre muestras de revestimiento extraídas in situ, de las cuales se cortaron 3 probetas de $15 \times 7 \mathrm{~cm}$ (PSP-1, PSP-2, PSP-3). El espesor de las placas es de $1,5 \mathrm{~cm}$.

El método elegido para medir el color es el sistema CIE $\mathrm{L}^{*} \mathrm{a}^{*} \mathrm{~b}^{*}$ ( $\mathrm{L}^{*}=$ luminosidad, $\mathrm{a}^{*}=$ coordenadas rojo/verde, $\mathrm{b}^{*}=$ coordenadas amarillo/azul), utilizando un colorímetro colorpen de la marca DR-Lange, con un área de medida circular de $6 \mathrm{~mm}$ de diámetro. Se parte de un inconveniente ya que por la naturaleza del material estudiado la superficie sobre la que se mide el color no es uniforme, por tanto se ha compensado la representatividad de la misma promediando un elevado número de mediciones (Figura 5), en cualquier caso, al tener todas las muestras el mismo tipo de superficie, la media comparativa obtenida a partir de 20 determinaciones permite minimizar esta circunstancia.

\subsection{Metodología de caracterización de las piezas tratadas con productos consolidantes}

Como complemento de los ensayos mecánicos se realizó un ensayo de resistencia superficial al arrancamiento, también llamado peeling test, que tiene por objeto comprobar in situ el grado de cohesión superficial del material de las placas de revestimiento. Este ensayo se realizó sobre cuatro placas de la fachada sur, por ser ésta la que mostraba un peor estado superficial de unión de los granos de las piezas. Se parte de la evidencia de que la fuerza cohesiva actual que tiene el material granular que conforma las placas es mínima, ya que con el simple roce de la mano se desprende con suma facilidad y el suelo del perímetro del templo se encuentra lleno de granos desprendidos. El ensayo se desarrolló mediante el diseño de un experimento del cual existe referencia por otros autores (20) (21), al igual que se hace en estudios similares ante la necesidad de evaluar propiedades en condiciones no normalizadas (7). Se aplica una cinta adhesiva de $20 \times 5 \mathrm{~cm}$ sobre la superficie, frotando sobre ella para garantizar una correcta fijación, posteriormente se tracciona de modo uniforme provocando el desprendimiento de los granos superficiales, que quedan adheridos a la cinta adhesiva, cuantificándose por pesada el material despegado.

Con los productos seleccionados a partir de la información suministrada por los análisis de los FTIR sobre la similitud de resinas actuales y la original se realizaron pruebas de capacidad consolidante. La elevada porosidad de las piezas y su sección $(1,5 \mathrm{~cm})$ permitía, a priori, presuponer una correcta penetración de los productos a través de la estructura de poros/huecos, en cualquier caso, como evaluación previa, se aplicaron las resinas sobre las probetas por pulverización hasta saturación superficial, pudiéndose comprobar la penetración alcanzada por el afloramiento de producto en la cara posterior de las probetas. Igualmente, con el fin de comprobar los tres productos se aplicaron en el laboratorio mediante pulverización en espray sobre marmolina limpia y suelta,

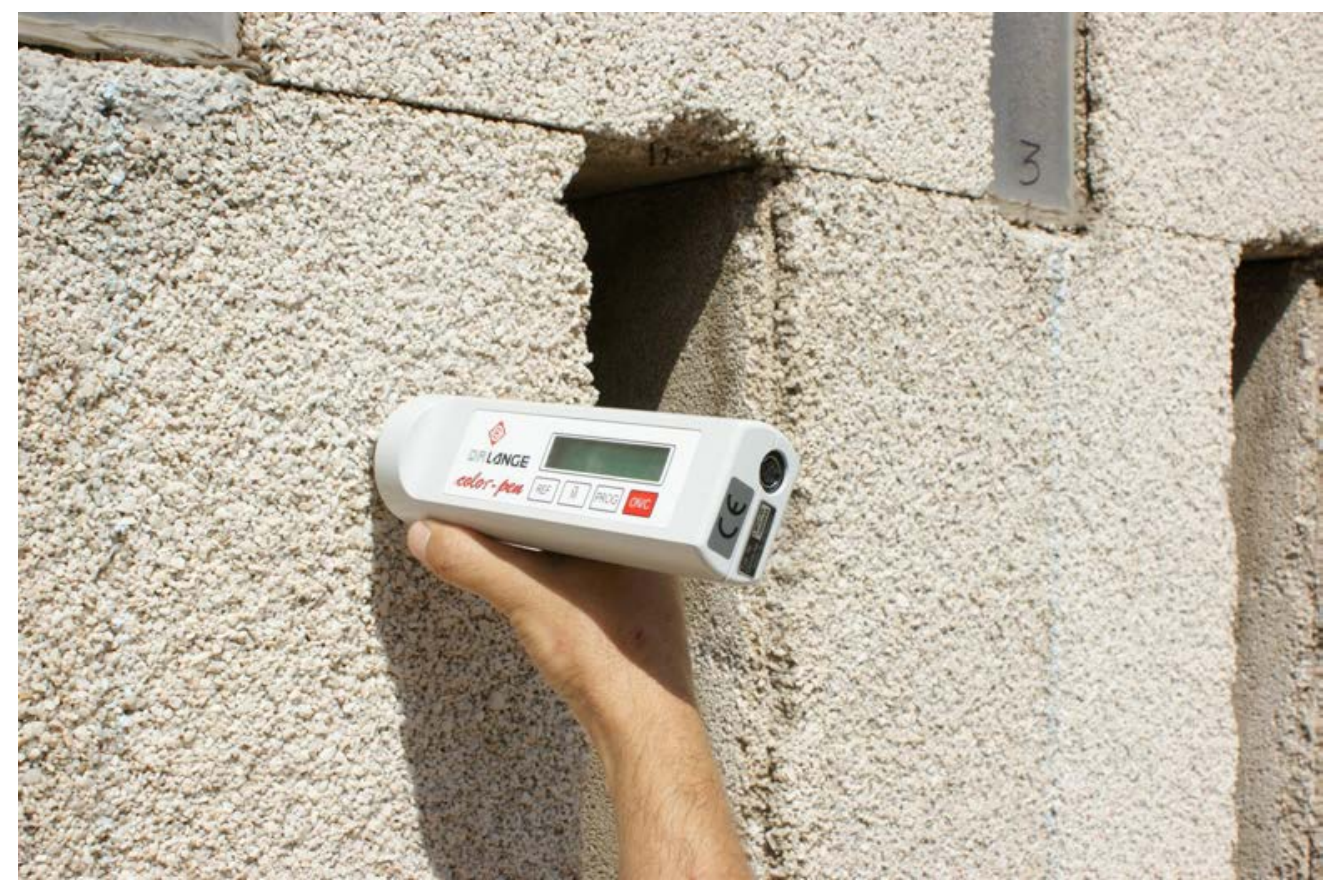

Figura 5. Determinación colorimétrica sobre placa de fachada. 
simulando una situación más desfavorable que la real. Estos ensayos preliminares de carácter organoléptico descartaron el Fluoline por su escaso poder consolidante en esta situación para este tipo de material.

Las placas seleccionadas para el estudio in situ de propiedades fueron tratadas hasta saturación superficial aparente con los tratamientos, que se aplicaron en la fachada mediante brocha (Figura 6). Las dosificaciones finales seleccionadas a partir de las fichas técnicas fueron Acril 33, diluido al 50 y $25 \%$ en agua, y Paraloid B-72, diluido al 10 y al $20 \%$ en acetona. Una vez transcurrido un período de polimerización de 30 días, se repitió el ensayo de resistencia superficial al arrancamiento para verificar el grado de fijación del árido (Figura 7).

Del mismo modo, transcurridos 30 días, se repitieron las medidas de color realizándose medidas colorimétricas sobre estas placas.

\section{ANÁLISIS DE RESULTADOS}

\subsection{Caracterización de las resinas}

El análisis de la resina utilizada en la fabricación de las placas puso de manifiesto que el contenido de polímero presenta actualmente una dosificación media del $0,67 \%$ en peso en las muestras estudiadas. El espectro de FTIR del concentrado del extracto muestra dos bandas que se pueden asignar a la presencia de grupos ésteres, ello da pie a pensar que la naturaleza de la resina original es de tipo poliéster alifático teniendo en cuenta que se encontraron bandas de dobles enlaces asociables a grupos alifáticos (5).

Con relación a los procesos de degradación, en el caso de las resinas tipo poliéster que se han detectado en el recubrimiento, el grupo éster es muy sensible a reacciones de hidrólisis, sobre todo en agua catalizada por ácidos. Por lo tanto un polímero de este tipo expuesto a la humedad y lluvia en combinación con la polución, que genera trazas de ácido en el agua, propiciará la hidrólisis de estos enlaces ésteres y la degradación del polímero (22). Adicionalmente, la presencia de grupos cromóforos, como los grupos carbonilo, favorece el desarrollo de reacciones provocadas por la acción de la luz solar (reacciones fotoquímicas) (23). Estas reacciones fotoquímicas propician la formación de radicales libres en el polímero, sobre todo en polímeros acrílicos, que pueden dar lugar a la ruptura de las cadenas. No parece que en el caso de estos polímeros la termodescomposición sea un factor determinante de su degradación (24) (25).

Los espectros de FTIR indicaron que las resinas Acrisil201, BA2028 y Epo15o tienen una naturaleza muy diferente a la resina utilizada en el revestimiento, mientras que las otras tres resinas (Acryl33, Fluoline y ParaloidB72) muestran espectros más similares al producto original, ya que son resinas acrílicas en las que el grupo éster está presente en su estructura y por tanto presentan bandas intensas similares. Sin embargo, como se ha indicado anteriormente, parece que la resina aplicada no es acrílica sino una resina de poliéster. No obstante, dadas las propiedades que muestran estas resinas respecto a su resistencia a radiaciones UV y a la humedad y su similitud estructural

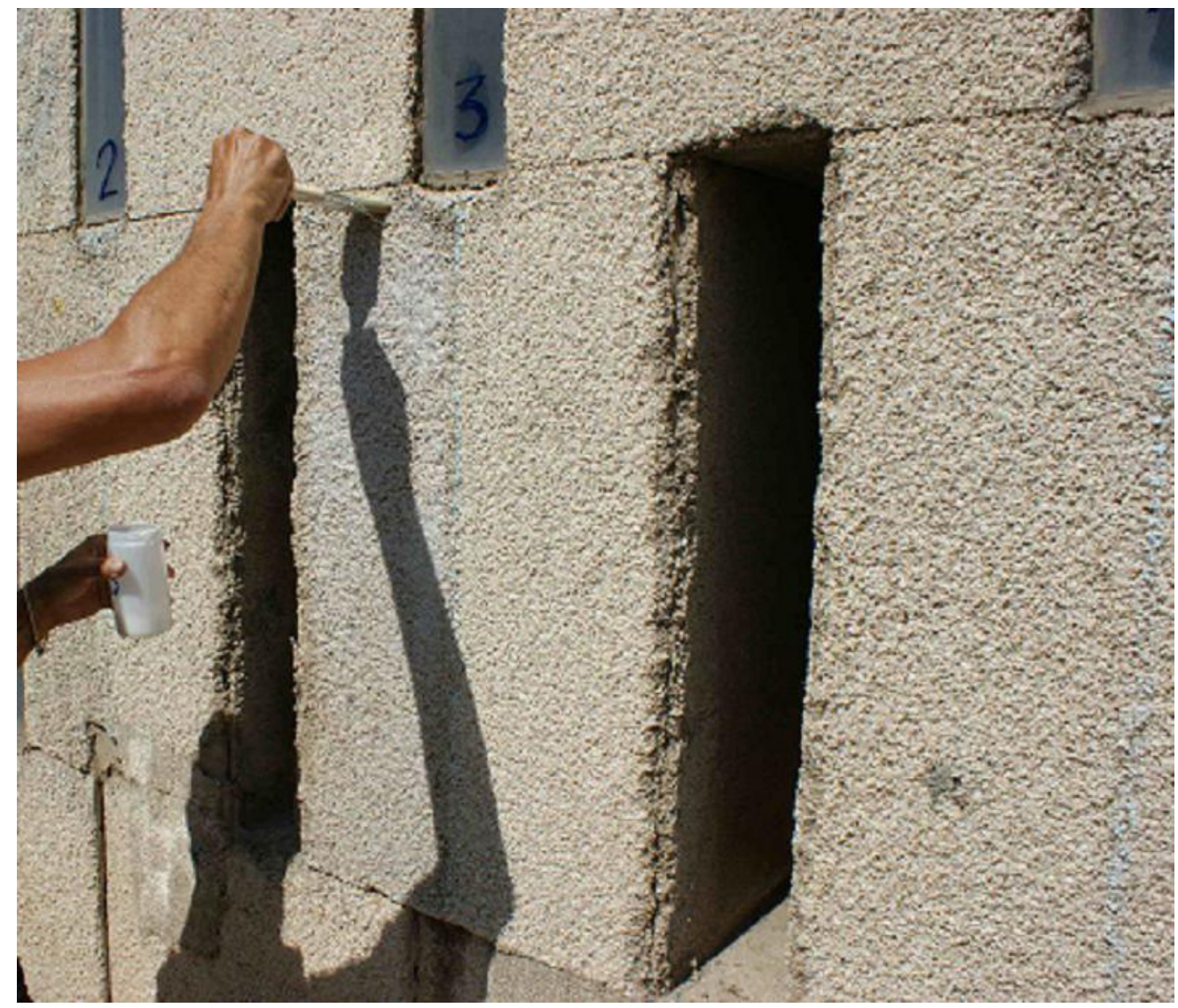

Figura 6. Aplicación de los tratamientos para los ensayos de evaluación de propiedades y comportamiento sobre el aplacado. 


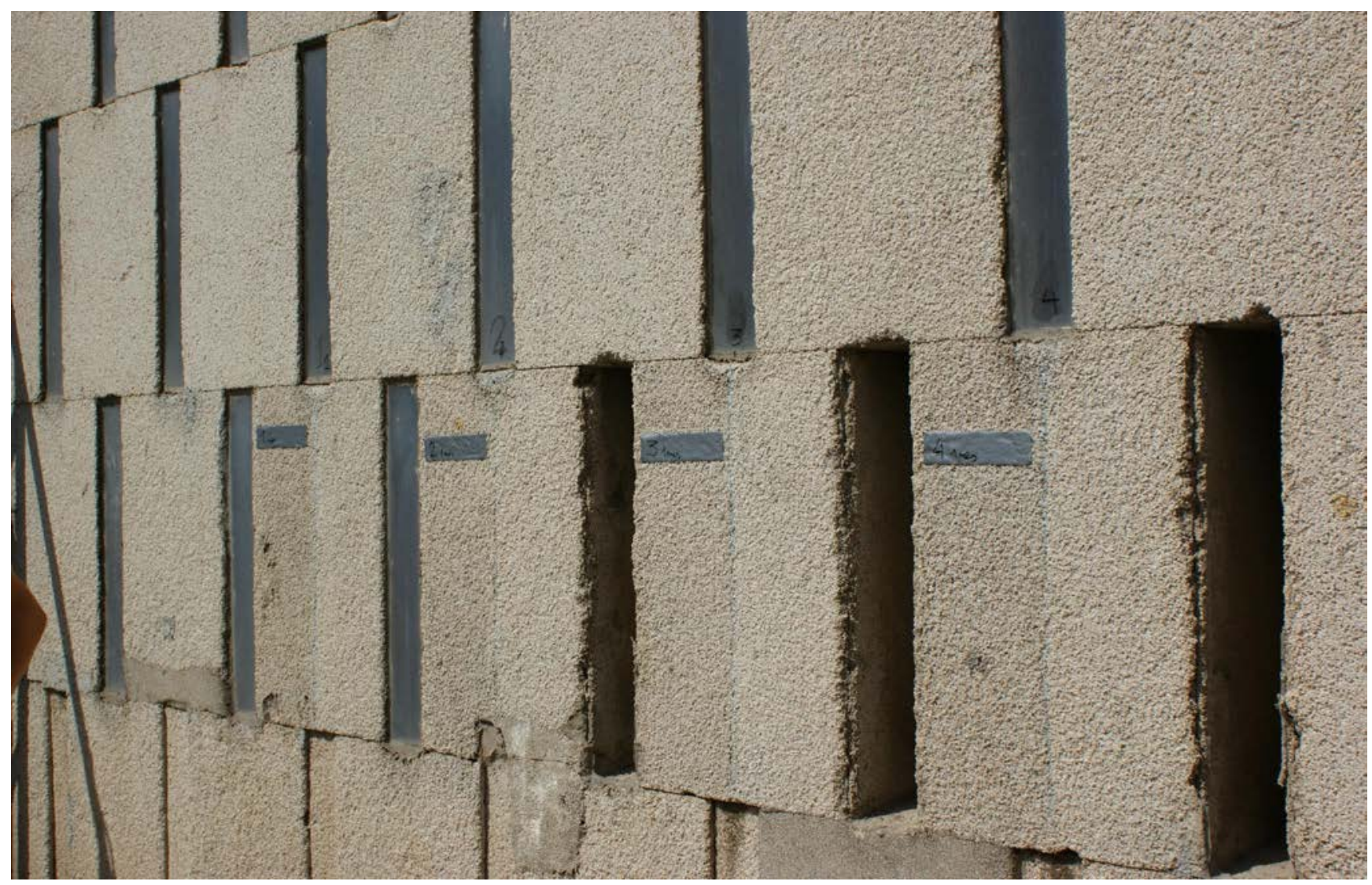

Figura 7. Ensayo de resistencia superficial al arrancamiento sobre placas de fachada.

Tabla 1. Cuadro resumen de la metodología utilizada para el análisis y evaluación de cada una de las características de las placas de revestimiento y de la efectividad de los tratamientos propuestos.

\begin{tabular}{|l|l|l|}
\hline \multirow{4}{*}{$\begin{array}{l}\text { Metodología de caracterización de las } \\
\text { piezas de revestimiento }\end{array}$} & Análisis de resinas originales & FTIR \\
\cline { 2 - 3 } & Árido & Análisis granulométrico \\
\cline { 2 - 3 } & Propiedades físicas & $\begin{array}{l}\text { Densidad aparente } \\
\text { Absorción } \\
\text { Porosidad accesible }\end{array}$ \\
\cline { 2 - 3 } & Caracterización mecánica & Resistencia a flexión de placas \\
\cline { 2 - 3 } $\begin{array}{l}\text { Metodología de caracterización de las } \\
\text { piezas tratadas con productos consolidantes }\end{array}$ & Colorimetría & CIE L*a*b* \\
\cline { 2 - 3 } & Caracterización mecánica & $\begin{array}{l}\text { Evaluación organoléptica de capacidad cohesiva } \\
\text { Ensayo de resistencia superficial al arrancamiento }\end{array}$ \\
\hline
\end{tabular}

respecto a la original, serían compatibles para ser aplicadas para la consolidación del recubrimiento estudiado.

\subsection{Caracterización del árido}

El árido está compuesto por un alto porcentaje de granos de un tamaño comprendido entre 2 y $4 \mathrm{~mm}$, próximo a un agregado unimodular (Figura 8). La curva granulométrica muestra que son prácticamente despreciables los tamaños inferiores, lo que confiere una elevada porosidad a las placas.

\subsection{Caracterización física}

Respecto a las propiedades físicas, cabe reseñar que tan sólo la densidad aparente calculada geométricamente aporta un valor relevante, puesto que la elevada porosidad impide el cálculo por determinación con pesada hidrostática en las muestras a vacío ya que el agua no queda retenida en los hue- cos por el elevado tamaño de éstos. El valor medio de densidad aparente obtenido fue de $1,48 \mathrm{~g} / \mathrm{cm}^{3}$. Los resultados se muestran en la Tabla 2.

Se puede observar como la densidad aparente determinada en las muestras a vacío/pesada hidrostática es mucho mayor a la calculada con geometría regular mediante volumen aparente. Esta diferencia se debe a la estructura macroporosa de las placas del revestimiento, que provocan que el agua ocluida durante la inmersión en los poros escape fácilmente por la acción de la gravedad cuando se extraen de este medio, generando la disminución del peso saturado de la muestra y distorsionando el valor de la densidad. Este efecto también se puede apreciar en los valores obtenidos para la absorción de agua y la porosidad, que son bastante bajos, y que sólo corresponden a los poros más pequeños del material, que son los que pueden retener absorbida el agua en su interior. 


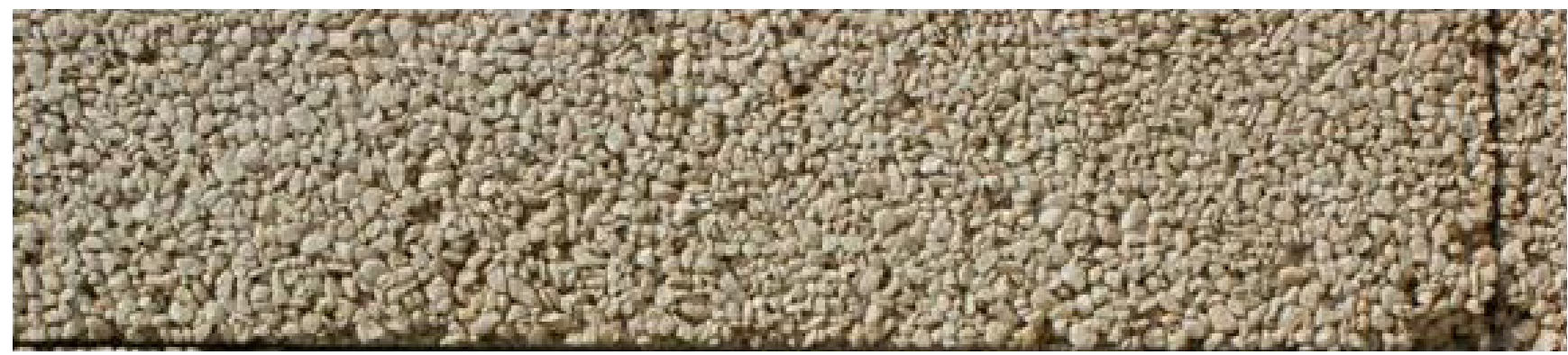

Figura 8. Imagen en la que se puede apreciar el aspecto de árido unimodular del agregado utilizado en la fabricación de las placas.

Tabla 2. Propiedades físicas de las placas.

\begin{tabular}{|c|c|c|c|c|}
\hline Muestra & $\begin{array}{c}\text { Densidad aparente } \\
\text { geométrica }\left(\mathrm{gr} / \mathrm{cm}^{3}\right)\end{array}$ & $\begin{array}{c}\text { Densidad aparente } \\
\text { a vacío }\left(\mathrm{g} / \mathrm{cm}^{3}\right)\end{array}$ & Absorción (\%) & Porosidad abierta (\%) \\
\hline PSP-1 & 1,45 & 2,35 & 4,26 & 5,70 \\
\hline PSP-2 II & 1,52 & 2,36 & 3,35 & 9,30 \\
\hline MEDIA & 1,48 & 2,35 & 3,80 & 7,50 \\
\hline
\end{tabular}

\subsection{Caracterización mecánica}

Los ensayos mecánicos de resistencia a flexión arrojaron valores muy bajos, casi despreciables, indicando que actualmente las placas solamente son capaces de resistir esfuerzos o cargas de flexión ínfimos (Figura 9). Hay que hacer notar que las placas no han sido concebidas para trabajar frente a dichas solicitudes (Tabla 3), lo que no es óbice para apuntar que la fragilidad que presentan ha propiciado su fracturación y desprendimientos parciales, siendo las temperaturas ambientales un factor muy influyente en este comportamiento (26) (10).

\subsection{Estudio de tratamientos de consolidación in situ}

El ensayo mecánico de arrancamiento se realizó in situ sobre cuatro placas distintas, comprobando la diferencia entre las placas en su estado inicial y tras la aplicación de los cuatro tratamientos de consolidación indicados anteriormente y sometidos a evaluación tras un período de polimerización de 30 días, con el objeto de comprobar la capacidad consolidante de los productos y la cohesión alcanzada en la superficie de las placas. En este caso se puso de manifiesto la necesidad de descartar el Paraloid-B72 como opción válida por tratarse de un producto en disolvente orgánico. Se observó que al tomar contacto con el aplacado, a pesar de mostrar capacidad consolidante (12), el disolvente provocaba la disolución de la resina original dando lugar al abombamiento y ligera deformación de las zonas tratadas (Figura 10).

Se puede comprobar la evidencia de mejora que supuso la aplicación de los consolidantes, en la Figura 11 se aprecian las cintas adheridas antes y después de la consolidación. No ha sido necesario realizar pesadas para expresar los resultados de forma cuantitativa, ya que ninguna de las cintas que se arrancaron sobre los cuatro consolidantes tuvo adherido ningún grano de marmolina.

\subsection{Caracterización colorimétrica}

En la Tabla 4 se exponen los resultados correspondientes a las coordenadas cromáticas correspondientes a las medias de 20 mediciones, llevadas a cabo sobre las placas en su estado inicial y al cabo de 30 días después de la aplicación de los cuatro tratamientos seleccionados.
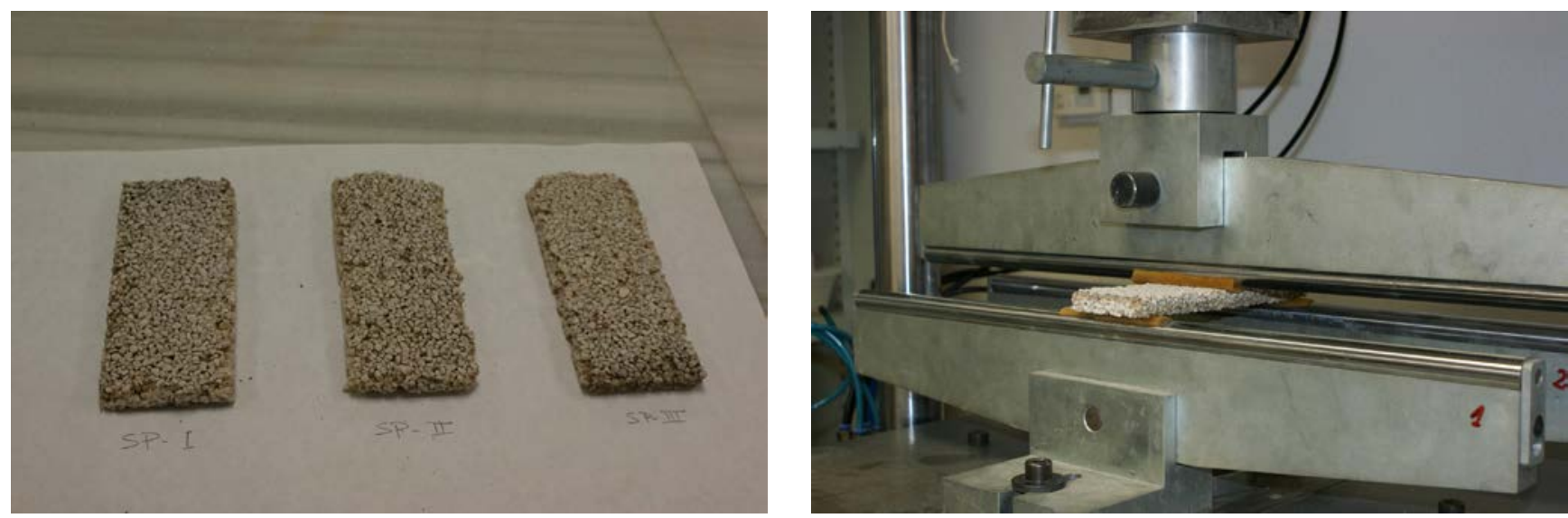

Figura 9. Imagen de las muestras de las placas de revestimiento y de una de las placas durante la rotura. 
Tabla 3. Resultados de los ensayos mecánicos.

\begin{tabular}{|c|c|c|c|}
\hline Muestra & $\begin{array}{c}\text { Dimensiones de las probetas } \\
\text { alto } \times \text { ancho }(\mathbf{c m})\end{array}$ & $\begin{array}{c}\text { Carga de rotura } \mathbf{F} \\
(\mathbf{K p})\end{array}$ & $\begin{array}{c}\text { Resistencia a flexión } \\
\left(\mathbf{N} / \mathbf{m m}^{\mathbf{2}}\right)\end{array}$ \\
\hline PSP-1 & Aprox. paralelepípeda $15,0 \times 7,1$ & 1 & 0,014 \\
\hline PSP-2 & Aprox. paralelepípeda $15,0 \times 7,0$ & 2 & 0,028 \\
\hline PSP-3 & Aprox. paralelepípeda $15,0 \times 7,0$ & 2 & 0,028 \\
\hline
\end{tabular}

Tabla 4. Coordenadas cromáticas de los revestimientos tratados y sin tratar.

\begin{tabular}{|l|c|c|c|c|c|c|}
\hline & \multicolumn{2}{|c|}{$\mathbf{L}$} & \multicolumn{2}{c|}{$\mathbf{a}$} & \multicolumn{2}{c|}{ b } \\
\cline { 2 - 6 } & $\mathbf{S} / \mathbf{T}^{*}$ & $\mathbf{T}^{* *}$ & $\mathbf{S} / \mathbf{T}$ & $\mathbf{T}$ & $\mathbf{S} / \mathbf{T}$ & $\mathbf{T}$ \\
\hline P1 (Paraloid 1,4) & 62,5 & 55,6 & 1,7 & 1,7 & 10,4 & 10,5 \\
\hline P2 (Paraloid 2,8) & 61,4 & 54,6 & 1,6 & 1,7 & 9,7 & 9,1 \\
\hline P3 (Acril 5) & 64,3 & 59,6 & 1,3 & 1,4 & 9,5 & 8,6 \\
\hline P4 (Acril 10) & 60,9 & 56,8 & 1,4 & 1,2 & 9,1 & 8,5 \\
\hline
\end{tabular}

* S/T medida realizada sin tratamiento, en el estado original de la placa.

** T medida realizada después de la aplicación de tratamiento y tras 1 mes de curado y polimerizado completo.

En la Tabla 5 aparecen los valores medios de las variaciones de estas tres coordenadas para cada tratamiento y cada tipo de probeta, junto con la variación de tono y el cambio de color global, que se calculan con las ecuaciones siguientes (27):

$$
\begin{aligned}
& \text { Variación de tono }=\sqrt{\Delta a^{2}+\Delta b^{2}} \\
& \Delta E=\sqrt{\Delta a^{2}+\Delta b^{2}+\Delta L^{2}}
\end{aligned}
$$

Este último valor es la distancia entre el punto que representa el color inicial de la placa y el punto que representa el color final del material, sin indicar hacia qué zona de la esfera de color se mueve el mismo. Un cambio global superior a 5 puede ser percibido por el ojo humano, aunque se consideran admisibles diferencias de hasta 10 (28).

Como se puede observar, los dos tratamientos con Acril han dado un cambio de color superior a 5, y los dos de Paraloid cercanos a 5 , lo que indica que pueden ser percibidos por el ojo humano, pero siempre inferiores a 10, por lo que la alteración se puede considerar como escasamente significativa.

\section{CONCLUSIONES}

El estudio de la tipología de resina utilizada originalmente en la fabricación de las placas ha aportado información suficiente para seleccionar los productos comerciales actuales para la restauración y consolidación de las mismas, de manera que se asegure la máxima compatibilidad de los polímeros. Así mismo, su cuantificación y el análisis granulométrico de la marmolina han permitido caracterizar compositivamente las placas.

Los ensayos físicos y mecánicos han sido el complemento necesario para llegar a una adecuada toma de decisiones respecto a la elección final del tratamiento a aplicar. En este capítulo el desarrollo de ensayos como el de arrancamiento, a similitud de otros empleados con otros materiales, se ha mostrado como una opción válida y que ha aportado información relevante para verificar el comportamiento de los consolidantes aplicados. El diseño de experimentos es un recurso que el técnico debe manejar como opción a la hora de evaluar propiedades que no tienen un marco normativo propio.
Tabla 5. Valores medios de las variaciones de coordenadas de las placas de revestimiento.

\begin{tabular}{|l|c|c|}
\hline & Variación de tono & Cambio de color global \\
\hline P1 & 0,01 & 6,90 \\
\hline P2 & 0,61 & 6,82 \\
\hline $\mathrm{P}_{3}$ & 0,91 & 4,79 \\
\hline $\mathrm{P}_{4}$ & 0,72 & 4,16 \\
\hline
\end{tabular}

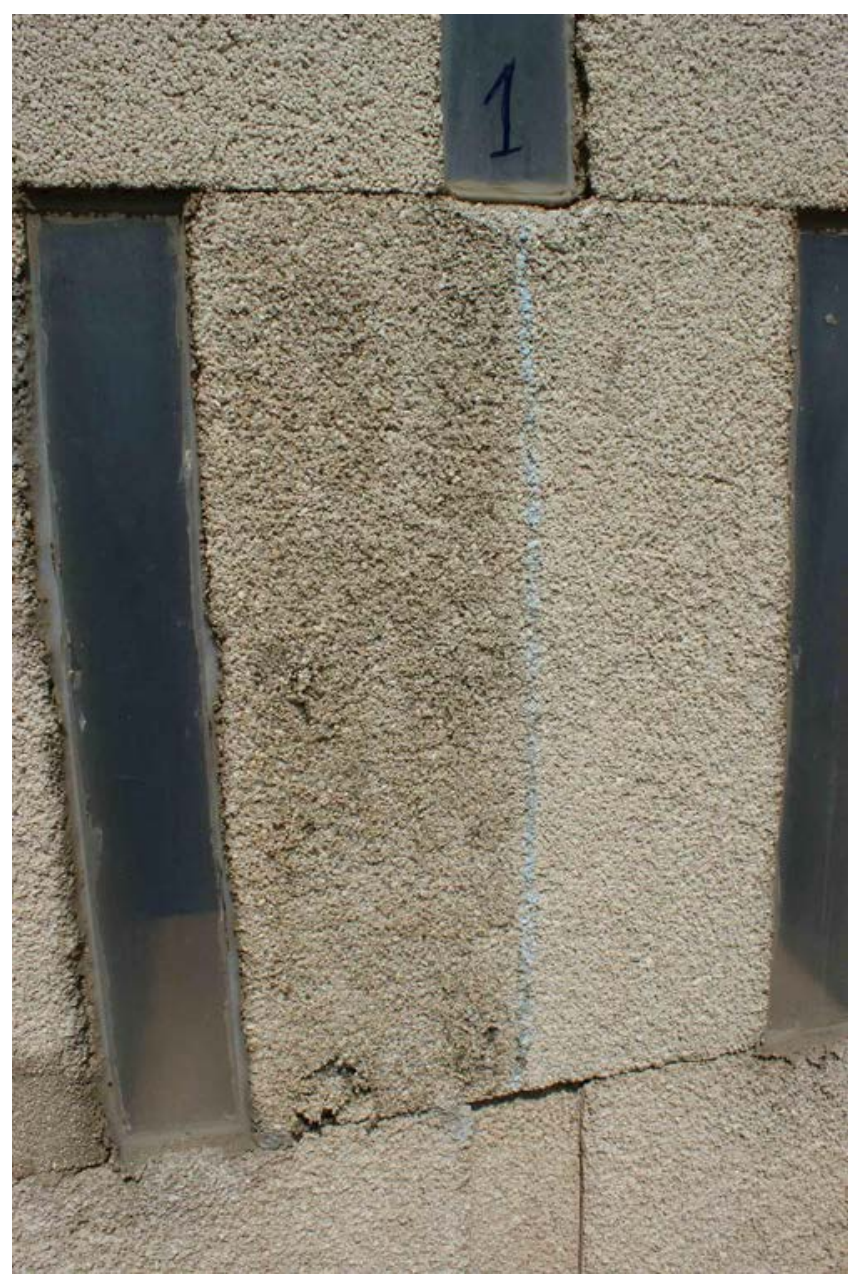

Figura 10. Se aprecia la ligera deformación debida al ataque del disolvente del tratamiento sobre la resina original, dando lugar en primera instancia a pequeños desprendimientos. 


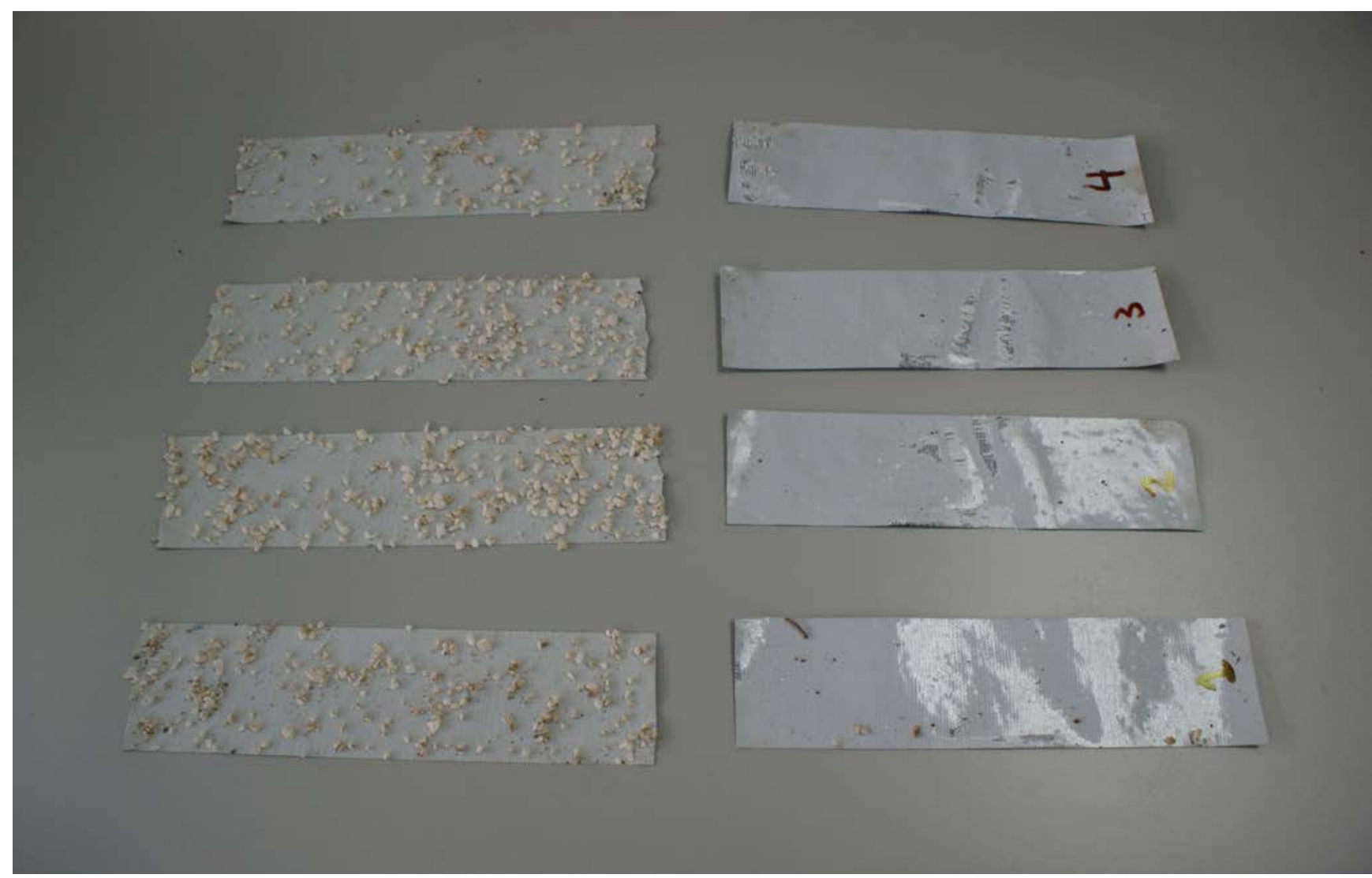

Figura 11. Imagen de las muestras de las placas de revestimiento y de una de las placas durante la rotura.

Las alteraciones cromáticas resultado de la aplicación de productos son consustanciales a una actuación de este tipo, ahora bien, se ha garantizado a través del control colorimétrico, atendiendo a las particularidades de la superficie analizada, que las modificaciones sufridas se han desarrollado siempre en un espectro visual aceptable.

\section{AGRADECIMIENTOS}

Los autores quieren dejar constancia de su agradecimiento al restaurador D. Carlos Núñez (Dédalo Bienes Culturales) y al Arzobispado de Sevilla por el interés mostrado en este trabajo y las facilidades para su realización.

\section{REFERENCIAS}

(1) Capilla Roncero. I., Sánchez-Cid, J. I., Ramos Carranza, A. (2003). Arquitectura del Racionalismo en Sevilla: inicios y continuidades. En II Seminario de la Arquitectura (pp. 20-34). Sevilla: FIDAS, COAS.

(2) Yáñez Sempere, J. L., García Torrente, U., Reina Fernández, J. C. (1992). Guía de la arquitectura de Sevilla y área metropolitana s. $X X$, Sevilla: Colegio Oficial de Arquitectos de Andalucía Occidental.

(3) López de Asiaín, J., Mosquera, E. (1996). El espíritu de la tercera generación en la arquitectura sevillana de los años 6o, Sevilla: ETS Arquitectura de Sevilla, 1996.

(4) http://www.iaph.es/arquitectura-contemporanea-andalucia/resumen.do?id=252466.

(5) Kamal, M. M., Tawfik, S. Y., Nosseir, M. H. (1987). Polyester mortar. Jour. Appl. Polymer Science, 33: 1609-1622, doi: http://dx.doi.org/10.1002/app.1987.070330516.

(6) Grassie, N., Marilyn, I. G. (1985). Degradation of epoxy polymers: 3-Photo-degradation of bisphenol-A diglycidyl ether. Polymer Degradation and Stability, 13(3): 249-259, doi: https://doi.org/10.1016/0141-3910(85)90012-6.

(7) Corpas, F. A., Codina, S., Ruiz Román, J. M., Ruiz Prieto, J. M., Alonso Santos. C. (2002). Characterization of material composite marble-polyester. Materiales de Construcción, 52(268): 65-71, doi: https://doi.org/10.3989/mc.2002.v52. i268.318.

(8) Ahmetli, G., Dag, M., Deveci, H., Kurbanli, R. (2011). Recycling Studies of Marble Processing Waste: Composites Based on Commercial Epoxy Resin. Jour. Appl. Polymer Science, 125(1), doi: https://doi.org/10.1002/app.34548 (online).

(9) Silva, M. A. G., Silva Z. C. G. (2007). Degradation of mechanical characteristics of some polymeric mortars due to aging. ACI Materials Jour., 104(4): 337-343, doi: https://doi.org/10.14359/18822.

(10) Silva, Z. C. G., Simao, J. (2007). Petrographic and mechanical aspects of accelerated ageing of polymeric mortars. Cement and Concrete Composites, 29(2): 146-156, doi: https://doi.org/10.1016/j.cemconcomp.2006.08.003.

(11) Tam, L., Lau D. (2015). Moisture effect on the mechanical and interfacial properties of epoxy-bonded material system: An atomistic and experimental investigation. Polymer, 57(28): 132-142, doi: https://doi.org/10.1016/j.polymer.2014.12.026. 
(12) Zoghlami, K., Gómez-Gras, D., Álvarez, A., De Luxán, M. P. (2005). Evaluation of consolidating and water repellent treatments applied to the miocene sandstone used in Tunisian Heritage Monuments. Materiales de Construcción 55(277): 25-39, doi: https://doi.org/10.3989/mc.2005.v55.i277.178.

(13) Villegas, R., Vale, F. J., Alcalde, M. (1991). Study of water-repellent treatments applied on limestone from Andalusian Cathedrals. Materiales de Construcción, 41(223): 19-27, doi: https://doi.org/10.3989/mc.1991.v41.i223.737.

(14) Luque, A., Cultrone, G., Sebastián, E., Cazalla, O. (2008). Effectiveness of stone treatments in enhancing the durability of bioclastic calcarenite in (Granada, Spain). Materiales de Construcción, 58(292): 115-128, doi: https://doi.org/10.3989/ mc.2008.41607.

(15) AENOR (1996). UNE-EN 933-2. Ensayo para determinar las propiedades geométricas de los áridos. Parte 2: Determinación de la granulometría de las partículas. Tamices de ensayo, tamaño nominal de las aberturas. Asociación Española de Normalización (AENOR).

(16) AENOR (2008). UNE-EN-13755:2008 Métodos de ensayo para piedra natural. Determinación de la absorción de agua a presión atmosférica. Asociación Española de Normalización (AENOR).

(17) AENOR (2007). UNE-EN-1936:2007 Métodos de ensayo para piedra natural. Determinación de la densidad real y aparente y de la porosidad abierta y total. Asociación Española de Normalización (AENOR).

(18) AENOR (2007). UNE-EN-12372:2006 Métodos de ensayo para piedra natural. Determinación de la resistencia a la flexión bajo carga concentrada. Asociación Española de Normalización (AENOR).

(19) Cerezo, A., Mas-Barber, X., Kröner, S. (2014). Study of the mechanical properties of mortars based on organic binders used in the reproduction of outdoor artworks. En Eur. Network for Conservation and Restoration Symp. Liège, Belgique.

(20) Drdácky, M., Lesák, J., Rescic, S., Slízkova, Z., Tiano, P., Valach, J. (2012). Standardization of peeling tests for assessing the cohesion and consolidation characteristics of historic stone surfaces. Materials and Structures, 45: 505-520, doi: https://doi.org/10.1617/s11527-011-9778-x.

(21) Espinosa-Gaitán, J., Martín-Chicano, A. (2016). A comparison of three methods of consolidation, for clacerous mixed stones. En Hughes, J., Howind, T. (Eds.). Science and Art: A Future for Stone: Proceedings of the 13th International Congress on the Deterioration and Conservation of Stone, Volume 2 (pp. 745-752). Paisley: University of the West of Scotland.

(22) Chércoles, R., San Andrés, M., De la Roja, J. M., Gómez, M. L. (2010). Factores responsables de la degradación química de polímeros. Efectos provocados por la radiación lumínica sobre algunos materiales utilizados en conservación. En 11. ${ }^{\text {a }}$ Jor. Conservación de Arte Contemp. (pp. 331-358). Madrid (España): Ed. Museo Nacional Reina Sofía.

(23) Doménech-Carbó, M. T., Silva, M. F., Aura-Castro, E., et al. (2011). Study of behaviour on simulated daylight ageing of artists' acrylic and poly(vinyl acetate) paint films. Anal and Bioanal Chem, 399: 2921-2937, doi: https://doi.org/10.1007/ s00216-010-4294-3.

(24) Pacheco-Torgal, F., Ding, Y., Jalali, S. (2012). Properties and durability of concrete containing polymeric wastes (tyre rubber and polyethylene terephthalate bottles): An overview. Const and Build Mat, 30: 714-724, doi: https://doi. org/10.1016/j.conbuildmat.2011.11.047.

(25) Saikia, N., De Brito, J. (2012). Use of plastic waste as aggregate in cement mortar and concrete preparation: A review. Const and Build Mat, 34: 385-401, doi: https://doi.org/10.1016/j.conbuildmat.2012.02.066.

(26) Ribeiro, M. C. S., Nóvoa, P. R., Ferreira, A. J. M., Marques, A. T. (2004). Flexural performance of polyester and epoxy polymer mortars under severe thermal conditions. Cement and Concrete Composites, 26(7): 803-809, doi: https://doi. org/10.1016/So958-9465(03)00162-8.

(27) Berns, R. S. (2000). Billmeyer and Saltzman's principles of color technology, New York, USA: Ed. Wiley-Interscience.

(28) Rodrigues, J. D., Grossi, A. (2007). Indicators and ratings for the compatibility assessment of conservation actions. Jour of Cult Heritage, 8: 32-43, doi: https://doi.org/10.1016/j.culher.2006.04.007. 